\title{
Blocking Infralimbic Basic Fibroblast Growth Factor (bFGF or FGF2) Facilitates Extinction of Drug Seeking After Cocaine Self-Administration
}

\author{
Madalyn Hafenbreidel ${ }^{1,2}$, Robert C Twining ${ }^{1,2}$, Carolynn Rafa Todd' and Devin Mueller, \\ 'Department of Psychology, University of Wisconsin-Milwaukee, Milwaukee, WI, USA
}

\begin{abstract}
Drug exposure results in structural and functional changes in brain regions that regulate reward and these changes may underlie the persistence of compulsive drug seeking and relapse. Neurotrophic factors, such as basic fibroblast growth factor (bFGF or FGF2), are necessary for neuronal survival, growth, and differentiation, and may contribute to these drug-induced changes. Following cocaine exposure, bFGF is increased in addiction-related brain regions, including the infralimbic medial prefrontal cortex (IL-mPFC). The IL-mPFC is necessary for extinction, but whether drug-induced overexpression of bFGF in this region affects extinction of drug seeking is unknown. Thus, we determined whether blocking bFGF in IL-mPFC would facilitate extinction following cocaine self-administration. Rats were trained to lever press for intravenous infusions of cocaine before extinction. Blocking bFGF in IL-mPFC before four extinction sessions resulted in facilitated extinction. In contrast, blocking bFGF alone was not sufficient to facilitate extinction, as blocking bFGF and returning rats to their home cage had no effect on subsequent extinction. Furthermore, bFGF protein expression increased in IL-mPFC following cocaine self-administration, an effect reversed by extinction. These results suggest that cocaine-induced overexpression of bFGF inhibits extinction, as blocking bFGF during extinction permits rapid extinction. Therefore, targeted reductions in bFGF during therapeutic interventions could enhance treatment outcomes for addiction.

Neuropsychopharmacology (2015) 40, 2907-29I5; doi:I0.I038/npp.20I5.I44; published online I0 June 2015
\end{abstract}

\section{INTRODUCTION}

Stimulant drug use results in structural and functional changes in reward-related brain regions (Flores and Stewart, 2000b; Pickens et al, 2011). For example, expansion of dendritic arbors on dopaminergic neurons in the ventral tegmental area (VTA) occurs following stimulant drug administration (Mueller et al, 2006), and both dendritic length and spine density are increased in the prefrontal cortex (PFC) and nucleus accumbens following prolonged withdrawal (Robinson and Kolb, 1997, 1999). Moreover, structural plasticity observed following drug exposure limits subsequent experiencedependent plasticity (Kolb et al, 2003). These long-lasting drug-induced morphological alterations are proposed to underlie specific characteristics of drug addiction, in particular the perseverance of compulsive drug seeking and relapse.

Basic fibroblast growth factor (bFGF or FGF2) is a neurotrophic factor that is necessary for survival, growth, and differentiation of neurons (Aoyagi et al, 1994; Wagner et al, 1999; Grothe et al, 2000). bFGF has been implicated in

\footnotetext{
*Correspondence: Dr D Mueller, Department of Psychology, University of Wisconsin-Milwaukee, 244I East Hartford Avenue, Garland Hall 224, Milwaukee, WI 532।I, USA, Tel: +4I4 229 6II3, Fax: +4I4 229 5219, E-mail: devinm@uwm.edu

${ }^{2}$ These authors contributed equally to this work.

Received 10 October 2014; revised 6 May 2015; accepted 15 May 2015; accepted article preview online 21 May 2015
}

the reorganization and dendritic growth of neurons after drug administration (Mueller et al, 2006), suggesting that bFGF contributes to the structural changes underlying the development and maintenance of drug abuse (Flores et al, 1998; Mueller et al, 2006). Indeed, endogenous bFGF in the VTA is required for the induction of locomotor sensitization by amphetamine (Flores et al, 2000a) and stimulant administration increases bFGF mRNA expression in the PFC (Fumagalli et al, 2006). Thus, the actions of bFGF may underlie the longlasting changes and perserveration associated with drug use and addiction.

Cues associated with a drug maintain addiction and promote relapse. Thus, reducing cue reactivity through extinction training, in which cues are no longer associated with the drug, could reduce relapse rates. The formation of a new extinction memory is dependent on the infralimbic region of the medial PFC (IL-mPFC). For example, inactivation of IL-mPFC disrupts extinction of drug seeking (LaLumiere et al, 2010) and inactivation of IL-mPFC after extinction results in reinstatement of drug seeking (Peters et al, 2008). Enhancing synaptic plasticity within IL-mPFC facilitates extinction of drug seeking (Gass et al, 2014) and some neurotrophic factors, such as brain-derived neurotrophic factor (BDNF), promote extinction-related plasticity in this region (Peters et al, 2010; Otis et al, 2014). However, the role of bFGF in IL-mPFC during extinction of drug seeking is unknown. Therefore, we used a self-administration 
model to determine the function of bFGF in IL-mPFC during extinction and how bFGF protein expression changes in IL-mPFC following extinction.

\section{MATERIALS AND METHODS}

\section{Subjects}

Male Long-Evans rats (Harlan Laboratories) weighing 250$300 \mathrm{~g}$ were housed and handled as previously described (Hafenbreidel et al, 2014). Rats had unlimited water access, but were food restricted (13-28 g rat chow/day) throughout experiments, except during surgery and recovery. Protocols were approved by the Institutional Animal Care and Use Committee at the University of Wisconsin-Milwaukee in accordance with National Institutes of Health guidelines.

\section{Drugs}

Cocaine $\mathrm{HCl}$ (National Institute of Drug Abuse) was dissolved in sterile $0.9 \%$ saline and administered intravenously (i.v.) at $0.25 \mathrm{mg} /$ infusion. Vehicle (IgG) or a neutralizing antibody against bFGF (anti-bFGF; Millipore, $05-117)$ was infused into IL-mPFC $(0.35 \mu \mathrm{g} / 0.35 \mu \mathrm{l} / \mathrm{side}$ at a rate of $0.15 \mu \mathrm{l} / \mathrm{min}) 1 \mathrm{~h}$ before extinction. This neutralizing antibody against bFGF blocks the biological function of bFGF, is highly selective for bFGF with no cross-reactivity to acidic FGF (aFGF or FGF1; Matsuzaki et al, 1989; Tao et al, 1997), and has been used previously (Flores et al, 2000a; Mueller et al, 2006).

\section{Self-Administration}

Sucrose, yoked-saline, and cocaine self-administration procedures were conducted using 12 sound-attenuated operant conditioning chambers (MED Associates) as previously described (Hafenbreidel et al, 2014). Briefly, 1 week after arrival, rats were food restricted for 3 days before a 10 -h sucrose-training session on a fixed ratio-1 (FR1) schedule of reinforcement. At the beginning of each session, the house light and right stimulus light turned on, and the right lever extended. Active lever presses resulted in delivery of a $20-\mathrm{mg}$ sucrose pellet. When criteria were met (100+ responses), sessions were shortened to 90 min on an FR1 schedule with a requirement of $50+$ responses/session.

Next, rats assigned to the yoked-saline or cocaine selfadministration conditions were anesthetized with ketamine/ xylazine $(87 / 13 \mathrm{mg} / \mathrm{kg}$, i.p.) and implanted with chronic intravenous catheters as previously described (Hafenbreidel et al, 2014). Rats in infusion experiments were also implanted with a 26-gauge double-barrel guide cannula aimed bilaterally at IL-mPFC (AP, +2.8, ML, $\pm 0.6, \mathrm{DV},-4.4 \mathrm{~mm}$ relative to bregma; see Otis et al, 2014 for details). Following surgery, rats were treated with penicillin $\mathrm{g}$ procaine $(75000$ units $/ 0.35 \mathrm{ml}$, s.c.) and carprofen $(5.0 \mathrm{mg} / 0.1 \mathrm{ml}$, s.c.). Following recovery, catheter patency was verified with $0.3 \mathrm{ml}$ of $1 \%$ Propofol (i.v.), a short-acting anesthetic that causes an immediate loss of muscle tone. Rats recovered for 7 days before behavioral testing. Catheter patency was maintained daily with heparinized saline $(\sim 0.2 \mathrm{cc}$ of $60 \mathrm{IU} / \mathrm{ml})$.

Rats in the cocaine self-administration condition were trained to self-administer cocaine (FR1) for 90-min daily sessions or until the progressive daily cap was achieved (2035 infusions/session). At the beginning of each session, the house light and right stimulus light turned on, and the right and left lever extended. When a rat pressed the active (right) lever, the stimulus light turned off for $20 \mathrm{~s}$ (time-out period), a tone $(65 \mathrm{~dB})$ sounded for $5 \mathrm{~s}$, and a 3-s cocaine infusion was initiated. All lever presses, reinforced or not, are reported as active lever presses during acquisition and extinction. Responding on the inactive lever was recorded, but had no programmed consequences. For experiment three, a yoked-saline control group was also included. These rats were yoked to a cocaine-administering rat throughout acquisition and extinction (if applicable), receiving identical cues and a saline infusion when the cocaine-administering rat pressed the active lever, but lever presses resulted in no programmed consequences. Rats were trained for 16-20 sessions. In order to achieve stable drug seeking, some rats $(n=28)$ received a priming infusion at the start of the session or received additional acquisition sessions. Groups were matched on the average number of infusions during selfadministration, the average number of infusions over the last 3 days of self-administration, and whether rats received priming injections or extra sessions.

To control for motor activity, a separate group of rats continued to lever press for sucrose (FR1) as during initial lever press training for 90-min daily sessions or until the progressive daily cap was achieved (50-95 presses/session). At the beginning of each session, the house light and right stimulus light turned on, and the right lever extended. Active lever presses resulted in delivery of a $20-\mathrm{mg}$ sucrose pellet. These rats did not receive catheters or cannula implants, but were trained on the same days as the cocaine and yokedsaline self-administration animals (experiment three). Groups were matched on average number of active lever presses.

\section{Experimental Manipulations}

Experiment one. The effect of neutralizing bFGF in IL-mPFC during extinction of cocaine seeking was examined. Following acquisition, rats were matched and assigned to groups, receiving either vehicle or anti-bFGF before the first 4 days of extinction. Before extinction, rats were adapted to microinfusion procedures. Injectors extending $1.0 \mathrm{~mm}$ past the guide cannula were lowered for $2 \mathrm{~min}$ on the first day. The next day, saline was infused at the same rate and volume as during drug manipulation, to allow the rats to adapt to changes in cranial pressure and mechanical stimulation induced by infusions. Next, vehicle or anti-bFGF was infused into IL-mPFC $1 \mathrm{~h}$ before four 30-min extinction sessions. Thirty-minute extinction sessions were used as previously described (LaLumiere et al, 2010; Hafenbreidel et al, 2014; Otis et al, 2014), to minimize extinction learning during the first four extinction sessions in order to better manipulate initial learning and determine whether antibFGF treatment facilitated subsequent extinction. Ninetyminute extinction sessions were used to test extinction retention from the 30-min sessions and measure subsequent extinction across days. Poor extinction memory retention was demonstrated by a significant increase in lever presses from day 4 to 5 , but good extinction memory retention was demonstrated by no change in lever pressing. Rats received 
a 2-day break for drug washout before extinction retention was tested during 90-min extinction sessions. During both 30- and 90-min extinction sessions, lever presses resulted in the same programmed consequences as during self-administration, but no cocaine was administered. Extinction was conducted in this manner to include both cues and behaviors to more closely model exposure therapy.

Experiment two. We next determined whether neutralizing bFGF in IL-mPFC in the absence of extinction training affected subsequent extinction. Following acquisition, rats were matched and assigned to two groups, receiving either vehicle or anti-bFGF. Rats were adapted to infusion procedures and were then infused for 4 days with either vehicle or anti-bFGF into IL-mPFC, and then immediately placed back in their home cages. Rats were then given a 2day break before being tested in daily 90-min extinction sessions.

To determine whether neutralizing bFGF in IL-mPFC altered general behavior, rats were infused with either vehicle or anti-bFGF $1 \mathrm{~h}$ before being placed in locomotor activity chambers and photobeam breaks were recorded for $20 \mathrm{~min}$.

Experiment three. To determine whether extinction altered IL-mPFC protein expression of bFGF or its primary receptor FGF receptor-1 (FGFR1; Lee et al, 1989; Reuss and von Bohlen und Halbach, 2003), we examined protein expression using western blottings following acquisition and extinction. Following acquisition, rats were matched and assigned to groups: extinction (coc-ext, yoked-sal-ext, and suc-ext) or no extinction (coc-noext, yoked-sal-noext, and suc-noext). Extinction of sucrose seeking, cocaine seeking, or yokedsaline administration was identical to acquisition, except that no sucrose, cocaine, or saline, respectively, was delivered following a lever press. A naive home-cage control group was also included that was neither manipulated nor handled (naive). Rats were given a 3-day break before coc-ext, yokedsal-ext, and suc-ext rats underwent daily 90-min extinction sessions. Coc-noext, yoked-sal-noext, and suc-noext rats remained in their home cages during these sessions. Following the last day of extinction, all groups (except naive) were tested for extinction retention with the same extinction procedure used throughout the experiment. Immediately after testing, rats were anesthetized with isoflurane gas and their brains were extracted and frozen at $-80^{\circ} \mathrm{C}$ for western blot analysis.

\section{Western Blottings}

To examine the effect of extinction on the protein expression of bFGF or FGFR1 in IL-mPFC, tissue was collected following extinction and was processed as previously described (Hafenbreidel et al, 2014; Fortress et al, 2015). Briefly, IL-mPFC was quickly dissected from frozen blocked tissue. Samples were stored at $-80^{\circ} \mathrm{C}$, re-suspended at $1: 25 \mathrm{w} / \mathrm{v}$ in lysis buffer, homogenized using a probe sonicator (Branson Sonifier 250), were electrophoresed on 18\% (for bFGF) or $7.5 \%$ (for FGR1) Tris-HCl gels (Bio-Rad), and transferred to PVDF membranes using a TransBlot Turbo system (Bio-Rad). Membranes were blocked in 5\% (for $\beta$ Actin or FGFR1) or $8 \%$ (for bFGF) milk for $1 \mathrm{~h}$ at room
Table I Average Number of Active and Inactive Lever Presses or Infusions Across the Last 3 Days of Self-Administration

\begin{tabular}{|c|c|c|c|c|}
\hline Experiment & Treatment & Active lever & Inactive lever & Infusions \\
\hline \multirow[t]{2}{*}{ I } & Veh (7) & $38.19 \pm 4.97$ & $8.10 \pm 4.86$ & $26.90 \pm 1.56$ \\
\hline & Anti-bFGF (8) & $36.75 \pm 2.77$ & $8.08 \pm 2.96$ & $27.86 \pm 0.99$ \\
\hline \multirow[t]{2}{*}{2} & Veh $(10)$ & $43.13 \pm 3.59$ & $1.83 \pm 0.63$ & $29.90 \pm 1.29$ \\
\hline & Anti-bFGF (I0) & $45.03 \pm 9.31$ & $9.60 \pm 8.61$ & $28.40 \pm 1.49$ \\
\hline \multirow[t]{7}{*}{3} & Naive (7) & - & - & - \\
\hline & Suc-NoExt (8) & $88 \pm 4.23$ & - & - \\
\hline & Suc-Ext (9) & $87 \pm 5.56$ & - & - \\
\hline & $\begin{array}{c}\text { Yoked-Sal-NoExt } \\
(12)\end{array}$ & $7 \pm 1.93$ & $3 \pm 0.71$ & - \\
\hline & $\begin{array}{l}\text { Yoked-Sal-Ext } \\
(\text { (II) }\end{array}$ & $5 \pm 1.72$ & $4 \pm 1.58$ & - \\
\hline & Coc-noext (12) & $38 \pm 4.08$ & $5 \pm 3.11$ & $28 \pm 1.90$ \\
\hline & Coc-ext (12) & $40 \pm 4.21$ & $\mid \pm 0.23$ & $28 \pm 1.45$ \\
\hline
\end{tabular}

Abbreviation: bFGF, basic fibroblast growth factor.

Numbers in parentheses are final group sizes.

temperature, followed by overnight incubation at $4{ }^{\circ} \mathrm{C}$ in primary antibodies for $\beta$-Actin (1:5000; Cell Signaling), bFGF (1:1000; Millipore), or FGFR1 (1:200; Santa Cruz, SC-121). Membranes were then incubated in secondary antibodies and developed using West Dura chemiluminescence (Pierce). Protein expression was imaged (ChemiDocMP gel imager, BioRad) and quantified by densitometry using Carestream software. To determine changes in protein expression, bFGF was normalized to $\beta$-Actin levels, which did not differ between groups ( $\beta$-Actin for bFGF normalization: $\mathrm{F}_{6,63}=1.708$, $P=0.134 ; \beta$-Actin for FGFR1 normalization: $\mathrm{F}_{6,63}=1.821$, $P=0.111)$. Data are expressed as percent immunoreactivity relative to controls.

\section{Data Analysis}

Lever presses and infusions during acquisition were analyzed by comparing the average of the last 3 days of cocaine selfadministration using $t$-tests. For extinction, lever presses were analyzed across days using a repeated-measures analysis of variance (ANOVA) or across days and between groups using two-way repeated-measures ANOVA. During the extinction retention test, lever presses were analyzed using $t$-tests. Some rats were removed from the analysis due to blocked or non-patent catheters $(n=5)$, sickness $(n=3)$, or not acquiring self-administration $(n=1)$. Final group sizes are reported in Table 1 and Figures. For locomotor activity, total photobeam breaks were analyzed using a $t$-test. For western blot analysis, mean optical densities were analyzed using a one-way ANOVA. All post-hoc tests were conducted, when appropriate, using Fisher's least significant difference test. Following behavioral procedures, verification of injector tip location was performed on cresyl violet-stained coronal sections.

\section{RESULTS}

\section{Neutralizing bFGF in IL-mPFC Facilitates Extinction}

To determine the effect of neutralizing bFGF in IL-mPFC on extinction of cocaine seeking, rats were infused with vehicle 
a

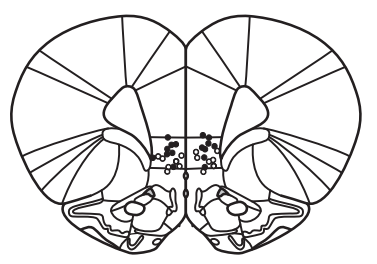

b

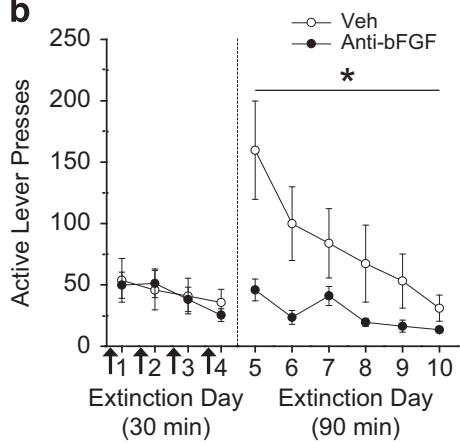

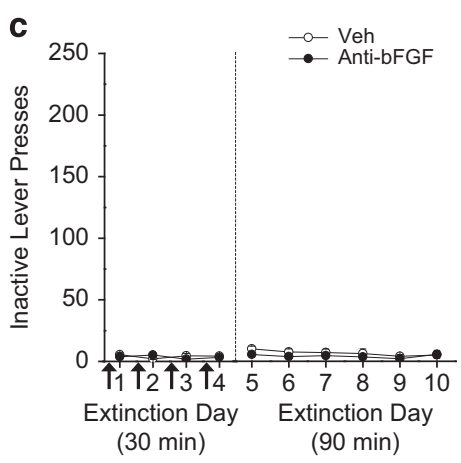

Figure I Neutralizing basic fibroblast growth factor (bFGF) in infralimbic medial prefrontal cortex (IL-mPFC) facilitates extinction. (a) Injector tip locations for vehicle (open circles) and anti-bFGF (closed circles) in IL-mPFC. (b) Pre-extinction session infusions of anti-bFGF into IL-mPFC (arrows) during the 30-min extinction sessions facilitated extinction retention compared with vehicle infusions. (c) Inactive lever presses during extinction. $* P<0.05$. Error bars are \pm SEM. Veh, $n=7$; anti-bFGF, $n=8$.

a

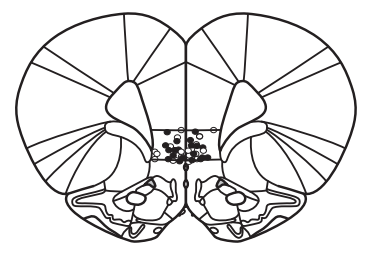

b

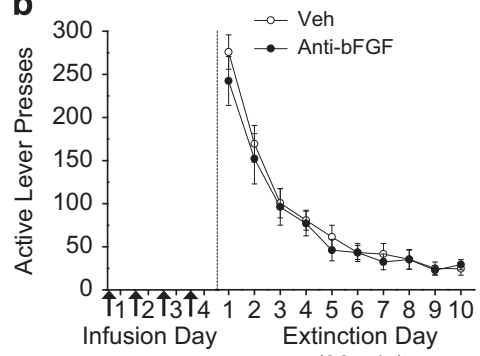

(90 $\mathrm{min})$

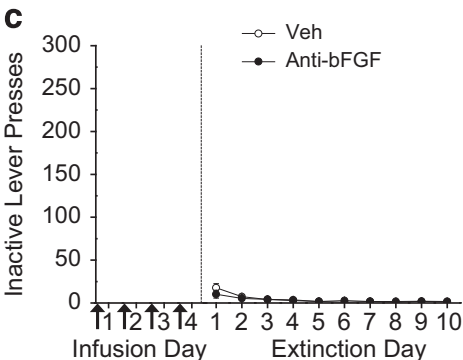

(90 $\mathrm{min})$

Figure 2 Neutralizing basic fibroblast growth factor (bFGF) in infralimbic medial prefrontal cortex (IL-mPFC) alone does not facilitate subsequent extinction. (a) Injector tip locations for vehicle (open circles) and anti-bFGF (closed circles) in IL-mPFC. (b) Infusions of either vehicle or anti-bFGF into IL-mPFC for 4 days (arrows), during which rats were returned immediately to their home cages, did not affect subsequent extinction. (c) Inactive lever presses during extinction. Error bars are \pm SEM. Veh, $n=10$; anti-bFGF, $n=10$.

or anti-bFGF before four 30-min extinction sessions (infusion sites depicted in Figure 1a). Extinction retention was tested on days 5-10 with $90 \mathrm{~min}$ drug-free extinction sessions. Lever presses and number of infusions were equivalent between groups across the average of the last 3 days of self-administration (Table 1). Overall, little extinction was observed across the first four 30-min extinction sessions (Figure 1b, left), but anti-bFGF-infused rats showed a robust reduction in lever pressing during the first 90-min extinction session compared with vehicleinfused rats (Figure $1 \mathrm{~b}$, right). Across the 30-min extinction sessions (days 1-4; active lever presses), ANOVA revealed no effect of day, treatment, or day by treatment interaction (all Fs $<1.20$, ps $>0.05$ ). For the 90 -min extinction sessions (days $5-10)$, ANOVA revealed a significant effect of day $\left(\mathrm{F}_{5,78}=\right.$ 4.297, $P=0.002)$ and treatment $\left(\mathrm{F}_{1,78}=25.130, P<0.0001\right)$, but no day by treatment interaction $\left(\mathrm{F}_{5,78}=1.572, P=0.178\right)$. These results indicate that both groups demonstrated extinction of lever pressing across days, but that neutralizing bFGF in IL-mPFC significantly reduced lever pressing compared with controls. Inactive lever presses (Figure 1c) did not differ across days or treatment regardless of extinction session duration ( $\mathrm{ps}>0.05$ ). Thus, neutralizing bFGF in IL-mPFC facilitated extinction.

\section{Neutralizing bFGF in IL-mPFC Alone is not Sufficient to Facilitate Extinction}

To determine whether neutralizing bFGF in IL-mPFC alone was sufficient to facilitate extinction, rats were infused with vehicle or anti-bFGF and immediately returned to their home cages for 4 days (infusion sites depicted in Figure 2a). Lever presses and number of infusions were equivalent between groups across the average of the last 3 days of selfadministration (Table 1). Overall, both groups extinguished across the 90-min extinction sessions (Figure 2b, right). For active lever presses, ANOVA revealed a significant effect of day $\left(\mathrm{F}_{9,180}=47.416, P<0.0001\right)$, but no effect of treatment or day by treatment interaction (all Fs $<1.37$, ps $>0.05$ ). For inactive lever presses (Figure 2c), ANOVA revealed a significant effect of day $\left(\mathrm{F}_{9,180}=9.398, P<0.0001\right)$, but no effect of treatment or day by treatment interaction (all Fs $>1.08$, ps $>0.05$ ). Thus, neutralizing bFGF in IL-mPFC without extinction training (immediately returning rats to their home cages) did not affect subsequent extinction. Therefore, extinction training in combination with bFGF neutralization in IL-mPFC is necessary to facilitate extinction.

To determine whether neutralizing bFGF in IL-mPFC affected general motor activity, rats were infused with vehicle or anti-bFGF $1 \mathrm{~h}$ before being placed in locomotor activity 
chambers for $20 \mathrm{~min}$. No differences in photobeam breaks between groups were observed $\left(t_{5}=0.565, P=0.597\right)$, demonstrating that locomotor activity was not affected by neutralizing bFGF in IL-mPFC (data not shown).

\section{Extinction Reduces Cocaine-Induced bFGF Protein Expression in IL-mPFC}

To determine whether endogenous bFGF expression was changed following extinction, bFGF protein expression was measured in IL-mPFC. Following acquisition, some rats (coc-ext, yoked-saline-ext, and suc-ext) underwent extinction, whereas other rats (coc-noext, yoked-saline-noext, and suc-noext) remained in their home cages. Lever presses and number of infusions (where applicable) were equivalent between sucrose, yoked-saline, or cocaine groups across the average of the last 3 days of self-administration (Table 1). For active lever presses during extinction of sucrose seeking (Figure 3a), ANOVA revealed a significant effect of day $\left(\mathrm{F}_{7,56}=34.561, P<0.0001\right)$, indicating that rats reduced lever presses across days. After the last day of extinction, both groups were tested for extinction retention (Figure 3b). For active lever presses, suc-ext rats pressed less than suc-noext rats $\left(t_{15}=-6.031, P<0.0001\right)$, demonstrating good extinction retention for the suc-ext group. Likewise, ANOVA revealed a significant effect of active lever presses in the cocext group $\left(\mathrm{F}_{7,77}=23.731, P<0.0001\right)$, indicating rats learned extinction (Figure 3c). For inactive lever presses (data not shown), ANOVA revealed a significant effect for coc-ext rats $\left(\mathrm{F}_{7,77}=9.660, P<0.0001\right)$. Comparing active and inactive lever presses across extinction days for coc-ext rats revealed a significant effect of lever $\left(F_{1,176}=154.796, P<0.0001\right)$, indicating that coc-ext rats lever pressed significantly more on the active lever than the inactive lever throughout (data not shown). Active (Figure 3e) and inactive (data not shown) lever presses in the yoked-saline group did not differ throughout extinction (active: $\mathrm{F}_{7,70}=1.446, P=0.201$; inactive: $\mathrm{F}_{7,70}=0.469, P=0.854$ ). Following the last day of extinction, all rats were tested for extinction retention. Cocext rats pressed less than coc-noext rats on active levers $\left(t_{22}=-3.208, P=0.004\right)$, demonstrating good extinction retention (Figure 3d). However, yoked-saline-ext rats and yoked-saline-noext rats did not differ on lever pressing $\left(t_{21}=-1.161, P=0.259\right.$; Figure $\left.3 \mathrm{f}\right)$, as expected. Inactive lever presses (data not shown) during the extinction retention test did not differ between coc-ext and coc-noext rats $\left(t_{22}=-1.981, P=0.060\right)$ or yoked-saline-ext and yokedsaline-noext rats $\left(t_{21}=1.267, P=0.219\right)$. Following the extinction retention test, rats were killed and IL-mPFC tissue was collected for analysis (Figure 4).

To determine whether extinction altered bFGF protein expression in IL-mPFC, we analyzed expression between groups. Figure 4a shows a representative area of tissue collection from IL-mPFC (bregma, $3.00 \mathrm{~mm}$; Paxinos and Watson, 2007), and Figure $4 \mathrm{~b}$ shows representative blots for each group. To determine whether natural reward reinforcement and extinction of reward seeking affected bFGF protein expression, tissue from naive, suc-noext, and suc-ext groups was compared. There were no significant differences in ILmPFC bFGF protein expression between naive, suc-noext, and suc-ext rats $\left(\mathrm{F}_{2,20}=0.003, P=0.997\right.$; Figure $\left.4 \mathrm{c}\right)$, indicating that neither sucrose reinforcement nor extinction of
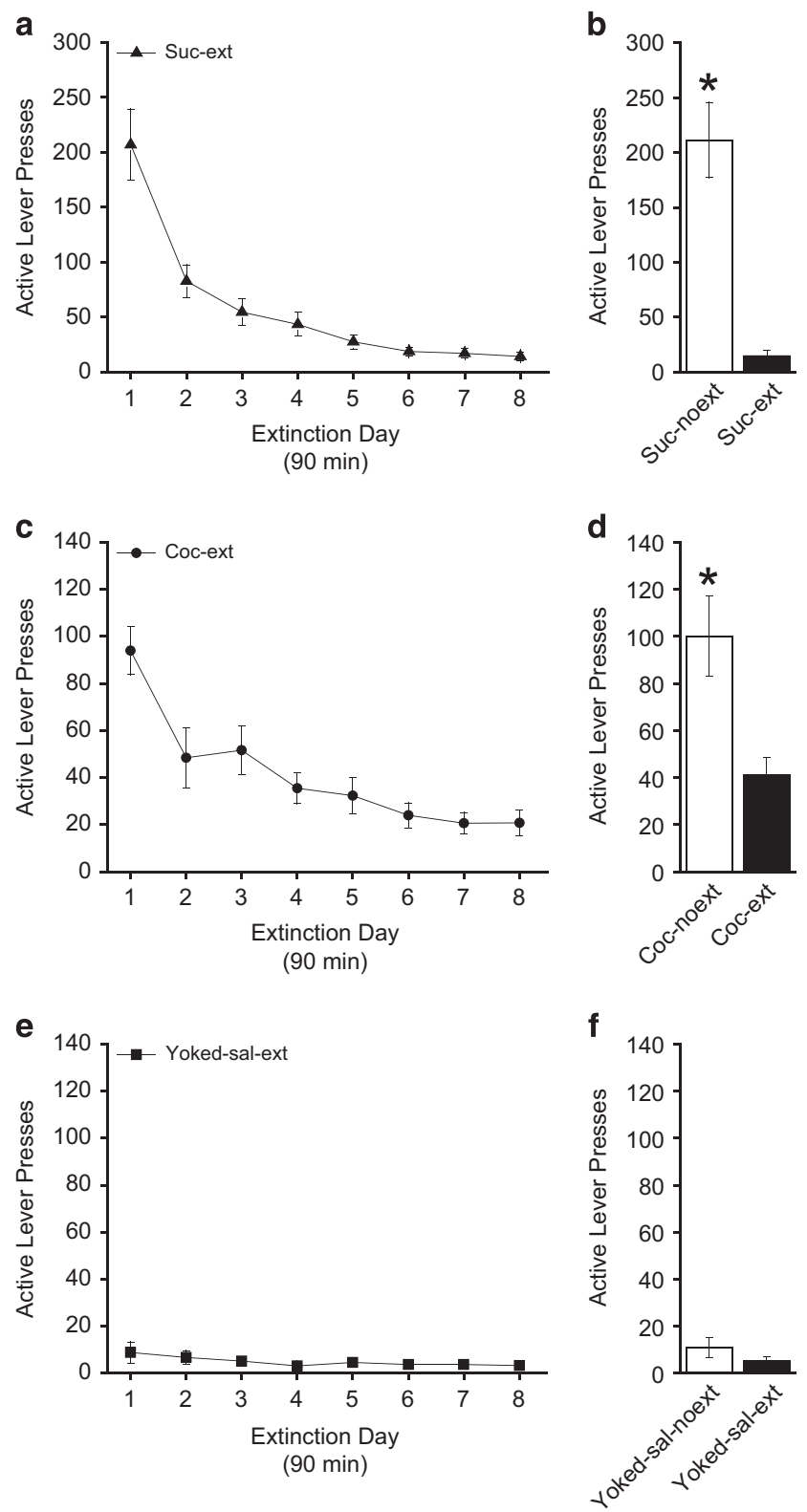

Figure 3 Extinction and extinction retention tests prior to protein analysis. Active lever presses during extinction of sucrose seeking (a) and during the extinction retention test (b). Active lever presses during extinction of cocaine seeking (c) and during the extinction retention test (d). Active lever presses during extinction following yoked-saline (e) and during the extinction retention test $(f)$. Error bars are \pm SEM. $* P<0.05$. Suc-noext, $n=8$; suc-ext, $n=9$; yoked-saline-noext, $n=12$; yoked-salineext, $n=11$; coc-noext, $n=12$; coc-ext, $n=12$.

sucrose seeking altered bFGF protein expression. Next, to determine whether cue presentation or i.v. infusions affected bFGF protein expression, tissue from naive, yoked-sal-noext, and yoked-sal-ext groups were compared. There were no significant differences in IL-mPFC bFGF protein expression between naive, yoked-sal-noext, and yoked-sal-ext rats $\left(\mathrm{F}_{2,27}=0.990, P=0.385\right.$; Figure $4 \mathrm{~d}$ inset $)$, indicating that cue presentation and i.v. infusions did not affect bFGF protein expression. Therefore, as these groups did not differ, they were collapsed into a single control group and 


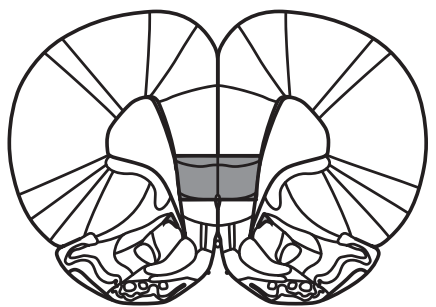

c

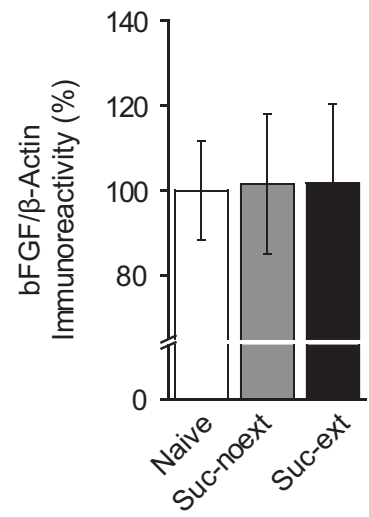

e

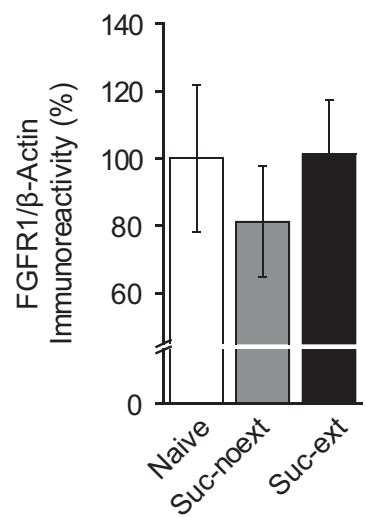

b

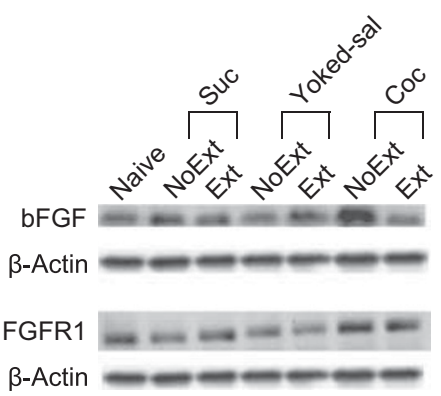

d
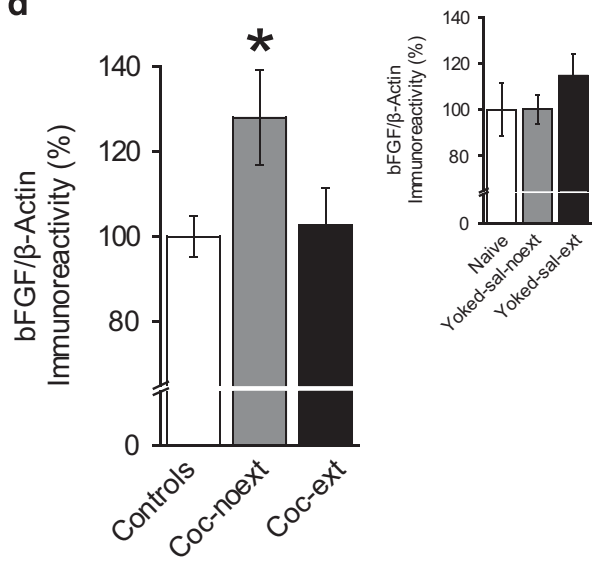

f

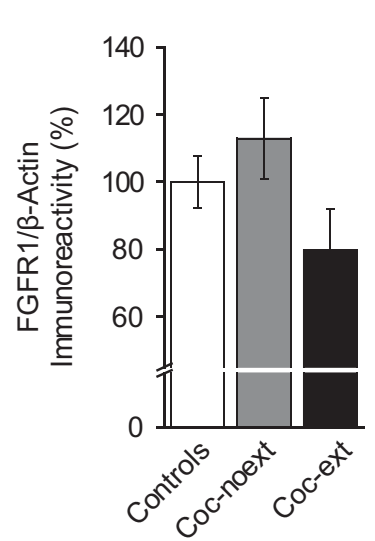

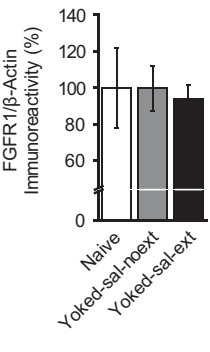

Figure 4 Cocaine self-administration increases basic fibroblast growth factor (bFGF) protein expression in infralimbic medial prefrontal cortex (IL-mPFC), an effect reversed by extinction. (a) Representative areas of tissue collection containing IL-mPFC. (b) Representative blots for each group. (c) bFGF protein expression was not altered between naive $(n=7)$, suc-noext $(n=8)$, or suc-ext $(n=8)$ groups. (d) bFGF protein expression was greater in coc-noext rats $(n=12)$ compared with coc-ext rats $(n=12)$ and controls $(n=30)$. (d, inset) Controls are shown separately. bFGF protein expression was not altered between naive $(n=7)$, yoked-sal-noext $(n=12)$, or yoked-sal-ext $(n=1 \mid)$ groups, and these groups were collapsed into a single control group. (e) FGF receptor-I (FGFRI) protein expression was not altered between naive $(n=7)$, suc-noext $(n=7)$, or suc-ext $(n=8)$ groups. $(f)$ FGFRI protein expression was not altered between controls $(n=28)$, coc-noext $(n=1 I)$, or coc-ext $(n=1 \mid I)$ groups. (f, inset) Controls are shown separately. FGFRI protein expression was not altered between naive $(n=7)$, yoked-sal-noext $(n=11)$, or yoked-sal-ext groups $(n=10)$, and these groups were collapsed into a single control group. * $P<0.05$. Error bars are \pm SEM.

compared with coc-noext and coc-ext (Figure 4d) to determine whether cocaine self-administration with or without extinction affected IL-mPFC bFGF protein expression. ANOVA revealed a significant difference in bFGF protein expression between control, coc-noext, and coc-ext rats $\left(\mathrm{F}_{2,51}=3.846, P=0.028\right)$. Post-hoc tests confirmed that coc-noext rats had significantly higher bFGF protein expression compared with control $(P=0.009)$ and coc-ext rats $(P=0.045)$, but control rats were not different from cocext rats $(P=0.797)$. Thus, only cocaine self-administration increased bFGF protein expression in IL-mPFC and this increase was reversed by extinction.

We next examined protein expression of the primary bFGF receptor, FGFR1, in IL-mPFC. There were no significant differences in FGFR1 protein expression between naive, suc-noext, and suc-ext rats $\left(F_{2,19}=0.369, P=0.696\right.$; 
Figure 4e), indicating that neither sucrose reinforcement nor extinction of sucrose seeking altered FGFR1 protein expression. There were also no significant differences in FGFR1 protein expression between naive, yoked-sal-noext, and yoked-sal-ext rats $\left(\mathrm{F}_{2,25}=0.075, P=0.928\right.$; Figure $4 \mathrm{f}$ inset), indicating that cue presentation and i.v. infusions did not affect FGFR1 protein expression. Therefore, these groups were collapsed into a single control group and compared with coc-noext and coc-ext rats (Figure 4d) to determine whether cocaine self-administration with or without extinction affected IL-mPFC FGFR1 protein expression. There were no significant differences in FGFR1 protein expression between control, coc-noext, and coc-ext rats $\left(\mathrm{F}_{2,47}=1.895\right.$, $P=0.162)$. Overall, FGFR1 protein expression in IL-mPFC was not changed in any condition.

\section{DISCUSSION}

We found that neutralizing bFGF in IL-mPFC during extinction rapidly facilitates extinction of cocaine seeking. This effect was dependent on extinction training, as neutralizing bFGF and immediately returning rats to their home cages without extinction training did not facilitate subsequent extinction. Moreover, cocaine self-administration increased endogenous bFGF protein expression in IL-mPFC, an effect reversed by extinction. However, protein expression of the primary receptor for bFGF, FGFR1, in IL-mPFC was not altered. Overall, these results indicate that blocking cocaine-induced increases in bFGF in IL-mPFC facilitates extinction of cocaine seeking, and that extinction can reduce bFGF protein expression in IL-mPFC.

Stimulant drugs, such as cocaine, induce structural and functional changes in specific brain regions, and these changes may underlie the perseveration of drug addiction. Following repeated cocaine injections or self-administration, increased numbers of dendritic spines and spine density are observed in reward-related brain regions such as the PFC (Robinson and Kolb, 1997, 1999; Robinson et al, 2001). Such structural changes could be regulated by neurotrophic factors, such as bFGF, as they promote neuron differentiation, neurogenesis, and experience-dependent plasticity (Aoyagi et al, 1994; Wagner et al, 1999; Flores and Stewart, 2000b). Following chronic or acute cocaine administration, bFGF mRNA expression is increased in reward-related brain regions such as the PFC (Fumagalli et al, 2006), and we showed that bFGF protein expression is similarly increased in IL-mPFC following cocaine self-administration. Interestingly, we did not see a significant change in FGFR1 protein expression, but did see a trend for reduced expression following extinction. FGFR1 is part of the tyrosine kinase receptor family (Johnson and Williams, 1993) and when bound by bFGF is trafficked to the nucleus (Myers et al, 2003; Zhang and Simons, 2014). Therefore, examining synaptic or nuclear expression instead of whole-cell expression may reveal a change in FGFR1 protein localization.

Previous research has identified the necessity of bFGF for amphetamine-induced sensitization (Flores et al, 2000a) and dendritic growth in dopaminergic neurons (Mueller et al, 2006), indicating that bFGF mediates maladaptive stimulantinduced alterations in neuronal function and structure. In addition, amphetamine or cocaine exposure impedes later life experience-dependent plasticity (Kolb et al, 2003) and neonatal injections of methamphetamine disrupt morris water maze learning in adulthood (Skelton et al, 2007), suggesting long-term adverse effects of stimulant use. bFGF inhibits both voltage-gated $\mathrm{Na}^{+}$(Hilborn et al, 1998) and $\mathrm{K}^{+}$ currents (Cuppini et al, 2009), suggesting that cocaineinduced overexpression of bFGF would functionally reduce intrinsic excitability of neurons. Importantly, reductions in intrinsic excitability can limit future learning (Sehgal et al, 2013). The IL-mPFC is necessary for extinction consolidation (Peters et al, 2008; LaLumiere et al, 2010; Otis et al, 2014) and enhancing IL-mPFC function facilitates extinction of drug seeking (LaLumiere et al, 2010; Gass et al, 2014; Otis et al, 2014). Here we show that neutralizing bFGF in IL-mPFC results in rapid extinction, suggesting that psychostimulantinduced overexpression of bFGF hinders extinction.

Our effects may be limited to extinction of drug seeking, as bFGF administration has been shown to enhance extinction of conditioned fear (Graham and Richardson, 2009, 2010a, b, 2011). However, stimulant administration itself increases bFGF expression (Fumagalli et al, 2006). Importantly, we found that sucrose self-administration and yoked-saline administration, with or without extinction, have no effect on bFGF protein expression in IL-mPFC. Thus, increases in bFGF expression in IL-mPFC are limited to cocaine selfadministration and are not due to operant responding, food reward, or cue presentation. Moreover, bFGF has opposing effects on extinction compared with another neurotrophic factor, BDNF. An infusion of BDNF into IL-mPFC facilitates extinction (Peters et al, 2010; Otis et al, 2014; Xin et al, 2014) and endogenous BDNF mRNA expression increases in ILmPFC during extinction of a conditioned taste aversion (Xin et al, 2014). Furthermore, reducing BDNF activity in the basolateral amygdala and dorsal hippocampus disrupts learning and extinction (Heldt et al, 2007; Sakata et al, 2013). In contrast, our results show that endogenous bFGF is reduced in IL-mPFC following extinction and blocking bFGF in this region facilitates extinction, suggesting that BDNF and bFGF have inverse roles during extinction. These opposing roles could be mediated by interactions with NMDA receptors. For example, BDNF facilitates extinction of drug seeking through its interaction with NMDA receptors (Otis et al, 2014). However, bFGF diminishes the function of these receptors. bFGF is neuroprotective (Mark et al, 1997; Flores and Stewart, 2000b) and cocaine exposure may generate a neuroprotective response. Cocaine blocks the reuptake of dopamine and the auto-oxidation of dopamine results in free-radical byproducts (Fornstedt et al, 1990). These free radicals recruit bFGF (Pechan et al, 1992), in particular in astrocytes (Gonzalez et al, 1995), which can protect neurons against oxidative stress (Hou et al, 1997). However, bFGF attenuates NMDA receptor function through interactions with calcium (Boxer et al, 1999). Consequently, the drug-induced overexpression of bFGF may impede extinction through reduction of NMDA receptor efficacy.

In conclusion, we demonstrate that neutralizing bFGF in IL-mPFC before extinction rapidly facilitates extinction, and that extinction of drug-seeking behavior reduces bFGF protein expression in IL-mPFC. To date, no research has been conducted concerning bFGF or FGFR1 and extinction following cocaine self-administration. Our results suggest a 
novel mechanism underlying the persistence of drug addiction. Therefore, understanding how drug use modifies bFGF could lead to the development of new psychotherapeutics to enhance therapeutic interventions, such as exposure therapy, for drug addiction.

\section{FUNDING AND DISCLOSURE}

The authors declare no conflict of interest.

\section{ACKNOWLEDGMENTS}

We thank John Schneider, Chad Smies, Jake Burkard, Jennifer Tuscher, Megha Sehgal, Dr James Otis, and Dr Ashley Fortress for technical assistance, and Dr Karyn Frick for generous use of equipment. This research was supported by R01 DA038042 and a grant from the University of Wisconsin-Milwaukee Graduate School to DM.

\section{REFERENCES}

Aoyagi A, Nishikawa K, Saito H, Abe K (1994). Characterization of basic fibroblast growth factor-mediated acceleration of axonal branching in cultured rat hippocampal neurons. Brain Res 661: 117-126.

Boxer AL, Moreno H, Rudy B, Ziff EB (1999). FGF-2 potentiates Ca (2+)-dependent inactivation of NMDA receptor currents in hippocampal neurons. J Neurophysiol 82: 3367-3377.

Cuppini C, Ambrogini P, Lattanzi D, Ciuffoli S, Cuppini R (2009). FGF2 modulates the voltage-dependent $\mathrm{K}+$ current and changes excitability of rat dentate gyrus granule cells. Neurosci Lett 462: 203-206.

Flores C, Rodaros D, Stewart J (1998). Long-lasting induction of astrocytic basic fibroblast growth factor by repeated injections of amphetamine: blockade by concurrent treatment with a glutamate antagonist. J Neurosci 18: 9547-9555.

Flores C, Samaha AN, Stewart J (2000a). Requirement of endogenous basic fibroblast growth factor for sensitization to amphetamine. J Neurosci 20: RC55.

Flores C, Stewart J (2000b). Basic fibroblast growth factor as a mediator of the effects of glutamate in the development of longlasting sensitization to stimulant drugs: studies in the rat. Psychopharmacology (Berl) 151: 152-165.

Fornstedt B, Pileblad E, Carlsson A (1990). In vivo autoxidation of dopamine in guinea pig striatum increases with age. J Neurochem 55: 655-659.

Fortress AM, Heisler JD, Frick KM (2015). The mTOR and canonical Wnt signaling pathways mediate the mnemonic effects of progesterone in the dorsal hippocampus. Hippocampus 25: 616-629.

Fumagalli F, Pasquale L, Racagni G, Riva MA (2006). Dynamic regulation of fibroblast growth factor 2 (FGF-2) gene expression in the rat brain following single and repeated cocaine administration. J Neurochem 96: 996-1004.

Gass JT, Trantham-Davidson H, Kassab AS, Glen WB Jr., Olive MF, Chandler LJ (2014). Enhancement of extinction learning attenuates ethanol-seeking behavior and alters plasticity in the prefrontal cortex. J Neurosci 34: 7562-7574.

Gonzalez AM, Berry M, Maher PA, Logan A, Baird A (1995). A comprehensive analysis of the distribution of FGF-2 and FGFR1 in the rat brain. Brain Res 701: 201-226.

Graham BM, Richardson R (2009). Acute systemic fibroblast growth factor-2 enhances long-term extinction of fear and reduces reinstatement in rats. Neuropsychopharmacology 34: $1875-1882$.
Graham BM, Richardson R (2010a). Early-life exposure to fibroblast growth factor-2 facilitates context-dependent long-term memory in developing rats. Behav Neurosci 124: 337-345.

Graham BM, Richardson R (2010b). Fibroblast growth factor-2 enhances extinction and reduces renewal of conditioned fear. Neuropsychopharmacology 35: 1348-1355.

Graham BM, Richardson R (2011). Intraamygdala infusion of fibroblast growth factor 2 enhances extinction and reduces renewal and reinstatement in adult rats. J Neurosci 31: 14151-14157.

Grothe C, Schulze A, Semkova I, Muller-Ostermeyer F, Rege A, Wewetzer K (2000). The high molecular weight fibroblast growth factor-2 isoforms $(21,000 \mathrm{~mol}$. wt and 23,000 mol. wt) mediate neurotrophic activity on rat embryonic mesencephalic dopaminergic neurons in vitro. Neuroscience 100: 73-86.

Hafenbreidel M, Rafa Todd C, Twining RC, Tuscher JJ, Mueller D (2014). Bidirectional effects of inhibiting or potentiating NMDA receptors on extinction after cocaine self-administration in rats. Psychopharmacology (Berl) 231: 4585-4594.

Heldt SA, Stanek L, Chhatwal JP, Ressler KJ (2007). Hippocampusspecific deletion of BDNF in adult mice impairs spatial memory and extinction of aversive memories. Mol Psychiatry 12: 656-670.

Hilborn MD, Vaillancourt RR, Rane SG (1998). Growth factor receptor tyrosine kinases acutely regulate neuronal sodium channels through the src signaling pathway. J Neurosci 18: 590-600.

Hou JG, Cohen G, Mytilineou C (1997). Basic fibroblast growth factor stimulation of glial cells protects dopamine neurons from 6-hydroxydopamine toxicity: involvement of the glutathione system. J Neurochem 69: 76-83.

Johnson DE, Williams LT (1993). Structural and functional diversity in the FGF receptor multigene family. Adv Cancer Res 60: 1-41.

Kolb B, Gorny G, Li Y, Samaha AN, Robinson TE (2003). Amphetamine or cocaine limits the ability of later experience to promote structural plasticity in the neocortex and nucleus accumbens. Proc Natl Acad Sci USA 100: 10523-10528.

LaLumiere RT, Niehoff KE, Kalivas PW (2010). The infralimbic cortex regulates the consolidation of extinction after cocaine self-administration. Learn Mem 17: 168-175.

Lee PL, Johnson DE, Cousens LS, Fried VA, Williams LT (1989). Purification and complementary DNA cloning of a receptor for basic fibroblast growth factor. Science 245: 57-60.

Mark RJ, Keller JN, Kruman I, Mattson MP (1997). Basic FGF attenuates amyloid beta-peptide-induced oxidative stress, mitochondrial dysfunction, and impairment of $\mathrm{Na}+\mathrm{K}+$-ATPase activity in hippocampal neurons. Brain Res 756: 205-214.

Matsuzaki K, Yoshitake Y, Matuo Y, Sasaki H, Nishikawa K (1989). Monoclonal antibodies against heparin-binding growth factor II/ basic fibroblast growth factor that block its biological activity: invalidity of the antibodies for tumor angiogenesis. Proc Natl Acad Sci USA 86: 9911-9915.

Mueller D, Chapman CA, Stewart J (2006). Amphetamine induces dendritic growth in ventral tegmental area dopaminergic neurons in vivo via basic fibroblast growth factor. Neuroscience 137: 727-735.

Myers JM, Martins GG, Ostrowski J, Stachowiak MK (2003). Nuclear trafficking of FGFR1: a role for the transmembrane domain. J Cell Biochem 88: 1273-1291.

Otis JM, Fitzgerald MK, Mueller D (2014). Infralimbic BDNF/TrkB enhancement of GluN2B currents facilitates extinction of a cocaine-conditioned place preference. J Neurosci 34: 6057-6064.

Paxinos G, Watson C (2007). The Rat Brain in Stereotaxic Coordinates, 6th edition edn. Elsevier.

Pechan PA, Chowdhury K, Seifert W (1992). Free radicals induce gene expression of NGF and bFGF in rat astrocyte culture. Neuroreport 3: 469-472.

Peters J, Dieppa-Perea LM, Melendez LM, Quirk GJ (2010). Induction of fear extinction with hippocampal-infralimbic BDNF. Science 328: 1288-1290. 
Peters J, LaLumiere RT, Kalivas PW (2008). Infralimbic prefrontal cortex is responsible for inhibiting cocaine seeking in extinguished rats. J Neurosci 28: 6046-6053.

Pickens CL, Airavaara M, Theberge F, Fanous S, Hope BT, Shaham Y (2011). Neurobiology of the incubation of drug craving. Trends Neurosci 34: 411-420.

Reuss B, von Bohlen und Halbach O (2003). Fibroblast growth factors and their receptors in the central nervous system. Cell Tissue Res 313: 139-157.

Robinson TE, Gorny G, Mitton E, Kolb B (2001). Cocaine selfadministration alters the morphology of dendrites and dendritic spines in the nucleus accumbens and neocortex. Synapse 39: 257-266.

Robinson TE, Kolb B (1997). Persistent structural modifications in nucleus accumbens and prefrontal cortex neurons produced by previous experience with amphetamine. J Neurosci 17: 8491-8497.

Robinson TE, Kolb B (1999). Alterations in the morphology of dendrites and dendritic spines in the nucleus accumbens and prefrontal cortex following repeated treatment with amphetamine or cocaine. Eur J Neurosci 11: 1598-1604.

Sakata K, Martinowich K, Woo NH, Schloesser RJ, Jimenez DV, Ji Y et al (2013). Role of activity-dependent BDNF expression in hippocampal-prefrontal cortical regulation of behavioral perseverance. Proc Natl Acad Sci USA 110: 15103-15108.

Sehgal M, Song C, Ehlers VL, Moyer JR Jr. (2013). Learning to learn - intrinsic plasticity as a metaplasticity mechanism for memory formation. Neurobiol Learn Mem 105: 186-199.

Skelton MR, Williams MT, Schaefer TL, Vorhees CV (2007). Neonatal (+)-methamphetamine increases brain derived neurotrophic factor, but not nerve growth factor, during treatment and results in long-term spatial learning deficits. Psychoneuroendocrinology 32: 734-745.

Tao Y, Black IB, DiCicco-Bloom E (1997). In vivo neurogenesis is inhibited by neutralizing antibodies to basic fibroblast growth factor. J Neurobiol 33: 289-296.

Wagner JP, Black IB, DiCicco-Bloom E (1999). Stimulation of neonatal and adult brain neurogenesis by subcutaneous injection of basic fibroblast growth factor. J Neurosci 19: 6006-6016.

Xin J, Ma L, Zhang TY, Yu H, Wang Y, Kong L et al (2014). Involvement of BDNF signaling transmission from basolateral amygdala to infralimbic prefrontal cortex in conditioned taste aversion extinction. J Neurosci 34: 7302-7313.

Zhang X, Simons M (2014). Receptor tyrosine kinases endocytosis in endothelium: biology and signaling. Arterioscler Thromb Vasc Biol 34: 1831-1837. 